\title{
Breast location of tuberculosis: a case report and literature review
}

\author{
Déhi Boston Mian" ${ }^{1 *}$, Védi André Serges Loué1, Aya Virginie Angoi ${ }^{1}$, Alexis Yao \\ Mohamed Kouyate ${ }^{2}$, Koffi N'guessan', Serge Boni'
}

\begin{abstract}
${ }^{1}$ Department of Gynecology and Obstetrics, University Hospital of Cocody, Abidjan, Côte d'Ivoire ${ }^{2}$ Department of Pathology, University Hospital of Treichville, Abidjan, Côte d'Ivoire
\end{abstract}

Received: 20 January 2022

Accepted: 08 February 2022

\section{*Correspondence:}

Dr. Déhi Boston Mian,

E-mail: bostondehimianlyahoo.fr

Copyright: () the author(s), publisher and licensee Medip Academy. This is an open-access article distributed under the terms of the Creative Commons Attribution Non-Commercial License, which permits unrestricted non-commercial use, distribution, and reproduction in any medium, provided the original work is properly cited.

\section{ABSTRACT}

Breast tuberculosis (TB) is a rare localization of extra-pulmonary TB. We report the first case observed in our daily activities to show the difficulties in the diagnosis and the good evolution in the treatment. In spite of the fact that it is rare, breast TB should not be ignored, especially in a country with high TB endemicity.

Keywords: TB, Breast, Histology, Anti-TB drugs, Literature review

\section{INTRODUCTION}

Tuberculosis (TB) is a chronic bacterial infection due to Mycobacterium tuberculosis. ${ }^{1}$ Globally, TB is now the number one killer infectious disease; more than $95 \%$ of TB deaths occur in low and middle-income countries (LMICs), where TB is among the top three causes of death for women aged 15 to $44 .^{2}$ Any organ can be affected by $\mathrm{TB}$, but the breast is an uncommon extrapulmonary TB site. ${ }^{3}$ The first case of breast TB was described by Cooper in 1829 as, "scrofulous swelling of the bosom of young women", but the first detailed description of the disease was not reported until the end of the $19^{\text {th }}$ century by Richet and Powers cited by Baharoon. ${ }^{3}$ It is generally believed that the infection of the breast is usually secondary to a primary site elsewhere in the body, which may or may not be clinically apparent; however, breast TB may be the primary site when no demonstrable tuberculous focus exists elsewhere., ${ }^{4,5}$ Lymphatic spread by retrograde extension from the axillary lymph nodes is considered the most common way the disease spreads. Propagation from cervical and mediastinal lymph nodes has occasionally been reported. ${ }^{3}$ There are no well-defined clinical features suggestive of breast TB. Because of its protean clinical presentations, establishing a diagnosis is difficult. For instance, it may be confused with breast carcinoma or pyogenic abscess. ${ }^{3,5}$ The diagnostic delay can last months, and patients often undergo numerous investigations and unsuccessful treatments before a definitive diagnosis is made. ${ }^{5,6}$ The most common clinical presentation is a lump, with or without a duct, painful or not. The lump can mimic carcinoma, being hard, with irregular borders, and fixed to either the skin or the muscle or even to the chest wall. ${ }^{5,6}$ Other presentations include diffuse breast swelling and edema, diffuse nodularity, nipple retraction, fistulation, multiple sinuses, skin ulcers, and recurrent abscess with or without axillary involvement. ${ }^{7}$

There are different ways to diagnose and follow up breast $\mathrm{TB}$, although none are ideal because of a combination of technical limitations and no or limited availability, particularly in LMICs. The gold standard for diagnosis is the detection of Mycobacterium tuberculosis by acid-fast bacteria Ziehl-Neelsen stain (AFB) or the isolation of the organism from the lesion on culture, but the former lacks sensitivity in paucibacillary samples, and the latter is relatively expensive and impractical in some low-resource settings. ${ }^{3}$ An alternative is polymerase chain reaction (PCR) to identify the Mycobacterium tuberculosis genetic material, but it is rarely used. ${ }^{8}$ Fine needle aspiration cytology (FNAC)-which detects the presence of epithelioid cell granulomas and necrosis-is often used instead, but has drawbacks-differential diagnosis is difficult in cases of granulomatous mastitis and sarcoidosis, for instance. ${ }^{8,9}$ Histopathology on biopsy 
identifies a chronic granulomatous inflammation (with caseous necrosis and Langhans-type giant cells). 5 Investigations such as ultrasonography, mammography, computed tomography, and magnetic resonance imaging do not give a conclusive diagnosis and, once again, are not widely available in LMICs. ${ }^{8,10}$ Treatment generally involves anti-TB medications with or without surgery. ${ }^{3,11}$ Medical-treatment often consists of an intensive four-drug, 2-month phase with isoniazid, rifampicin, pyrazinamide, and ethambutol, followed by a two-drug, 6-month (or longer) continuation phase with isoniazid and rifampicin.

Despite several published literature reviews of breast TB, this was the first case reported in our less and mid developed country; therefore, we aimed reviewing the literature about risk factors and clinical, diagnostic, and therapeutic aspects of breast TB in women. ${ }^{9-11}$

\section{CASE REPORT}

Ms. D M, 43 years old, is the $4^{\text {th }}$ gestation and second parturition. She has been correctly vaccinated against TB during her childhood. No history of TB contact, previous medical history of treated TB and family history of pulmonary TB were related. Both lump and mastalgia were the presenting symptoms. The clinical examination revealed a weight loss of more than $10 \%$ of body weight (from 60 to 50 kilogram). The lumps were located in the upper outer quadrant of the left breast. The mean size of the lump was $3 \times 3 \mathrm{~cm}$. The lump was irregular, mobile and painful. Ipsilateral axillary lymphadenopathy was noted with no breast abscess. Associated with breast (Figure 1). $\mathrm{TB}$, other foci of TB were represented by cervical lymph node TB (Figure 2). Tuberculin skin test was positive and the patient was tested negative for human immunodeficiency virus. Mammography, ultrasound and excision biopsy were requested but not performed. The evolution is marked, two months later, by appearance of a cutaneous fistula of left breast lump associated with ipsilateral axillary and cervical lymphadenopathy. Furthermore, there was a lipoma in front of the left pectoral muscle of about $4 \mathrm{~cm}$ of mean size (Figure 1). Excision biopsy was immediately performed in order to exclude a possible breast cancer. The diagnosis was confirmed based on the presence of epithelioid cell granulomas and caseous necrosis (Figure 3). The detection of Mycobacterium tuberculosis in smear or culture for confirmed the diagnosis was not realized (Figure 3). Patient received antitubercular therapy for a mean duration of 09 months. Type of antitubercular treatment included Two months of isoniazid, rifampicin, pyrazinamide, and ethambutol +4 months of isoniazid and rifampicin. The disease evolution was a recovery of weight with $10 \mathrm{~kg}$ gained, disappearance of the breast lump, a decreased mean size of the axillary and cervical lymph nodes. The persistence of the lymph nodes at the $6^{\text {th }}$ month obliged to prolonged treatment for 3 months combining $150 \mathrm{mg}$ of rifampicin and $75 \mathrm{mg}$ of isoniazid. The clinical healing was completed after 9 months of treatment before the disappearance of all clinical signs and a normal mammography and ultrasound results.

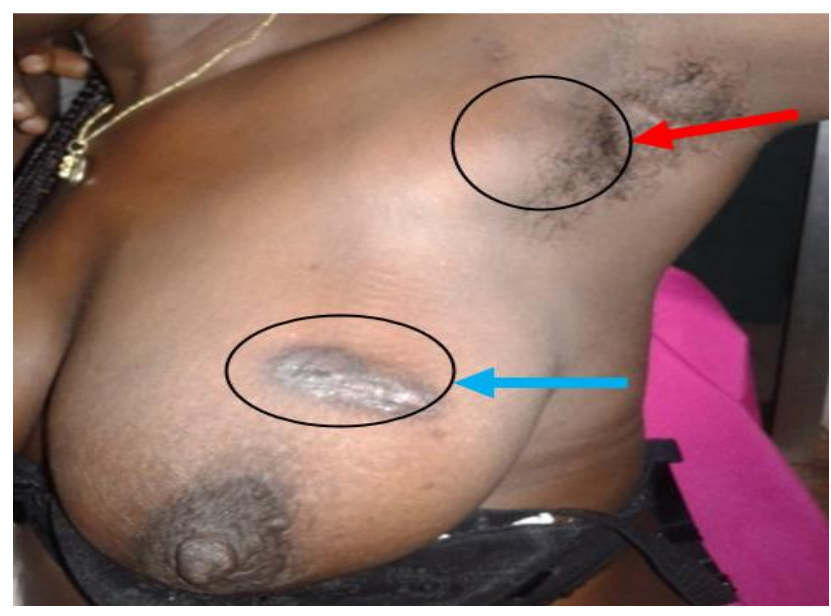

Figure 1: Axillary lymph node shows (red arrow), 2breast fistula (blue arrow).

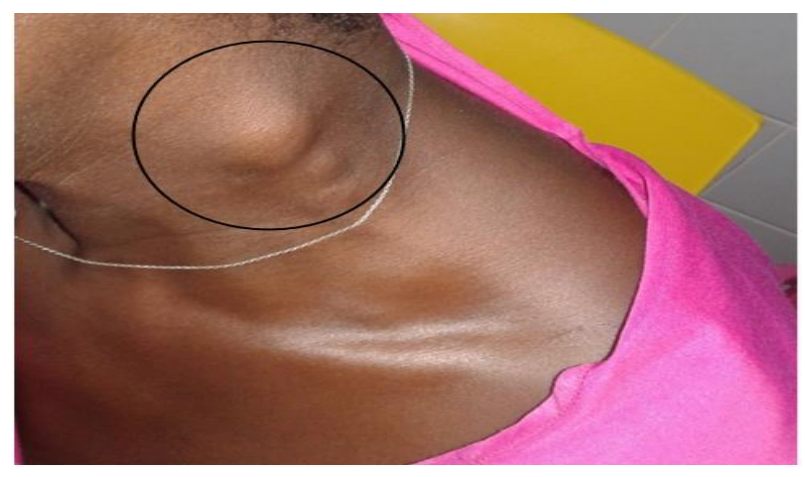

Figure 2: Cervical lymph nodes.

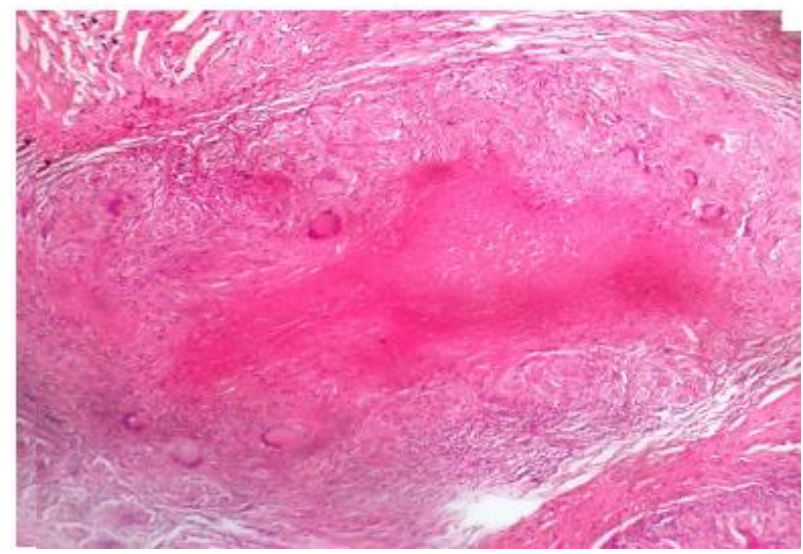

Figure 3: Epithelial giant cell granuloma centered by a structureless necrosis (HE X 20).

After micro biopsy, the histological examination concluded at a caseum follicular tuberculous mastitis with the presence of a giant epithelial cells granuloma, sometimes centered on caseous necrosis. The granuloma consisted of epithelioid cells, giant Langham's cells, lymphocytes and plasma cells as shown in Figure 3. 


\section{DISCUSSION}

Tubercular infection is prevalent all over the world with higher incidence reported in the less-developing countries. $^{12}$ Breast TB remains a very rare disease, representing $0.2 \%$ of extrapulmonary TB cases. ${ }^{11-14} \mathrm{We}$ reported the first case of breast TB observed in many years of gyanecology pratice to aim the diagnosis and management difficulties in a less and mid developped country. The average reported prevalence was $1.7 \%$, ranging from $0.2 \% 20$ to $6.8 \% .{ }^{10}$ In a first-time systematic review of literature, 1,478 cases of breast TB have been identified throughout risk factors and clinical, diagnostic, and treatment aspects. ${ }^{15}$ The prevalence in Western countries is less than $0.1 \% .^{2,5}$ Breast TB more commonly affects women of childbearing age (average age 29 years)..$^{15}$ Elderly women may also be affected as we reported in case, whereas the disease is very rare under the age of $18 . .^{11,16,17}$

As the breasts of multiparous and lactating females are more sensitive to infection and trauma, tubercular mastitis is more common among them as confirmed in reported case. Incidence of involvement of the right and left breast is equal, except in one study in which there is a slight higher tendency for the right breast to be affected. ${ }^{11,15}$ In case we described a left location of breast node in a 43 years old pauciparous women. TB of the male breast is an extremely rare condition. ${ }^{7,16}$ Lilleng et al in a study of 809 cases of male breast mass, did not find a single case of TB. ${ }^{7}$ In pregnant and lactating women, the increased vascularity of the breast with dilated ducts predispose to infection. ${ }^{17}$ Pregnancy suppresses the T-helper 1 pro-inflammatory response, which may increase susceptibility to a new infection or reactivation of $\mathrm{TB} .{ }^{17}$ It is difficult to understand whether this might be the case: on the one hand, a small percentage were either pregnant or lactating, and on the other hand, more than $70 \%$ were multiparous, and, considering the long diagnostic delays, breast TB might have been triggered by a previous pregnancy. ${ }^{15} \mathrm{HIV}$ infection carries an increased risk for primary TB, for reactivation of previous $\mathrm{TB}$, and for second episodes of TB from exogenous reinfection which was not found in our case. ${ }^{18,19}$

Usually, the duration of symptoms ranged from 6 months to 2 years. Chest wall TB simulating breast carcinoma: imaging appearance. ${ }^{8,20}$ Breast $\mathrm{TB}$ as a presenting manifestation of HIV is extremely rare as we observed in case. ${ }^{18,19}$ The HIV status was described in 211 cases, and approximately one in five were positive. ${ }^{15}$ The clinical presentation of breast TB is generally poorly described in the literature, and clinically important features are not uniformly reported or not reported at all. ${ }^{15}$ The average duration of symptoms before diagnosis is highly variable, spanning from a few weeks in Europe to more than 7 months in India and sub-Saharan Africa. ${ }^{1,3,11,21,22}$ This includes both patient and health system delays, and reflects the range of cultural, psychological, and economic components, as well as the diagnostic challenges. The disease is generally mono-lateral and can equally affect either breast as in case. ${ }^{13,23}$

A lump is the most common presentation, with other less common forms being cold abscess and diffuse breast inflammation. ${ }^{15,24}$ Breast lumps are mostly misdiagnosed as fibroadenoma, malignancy, or breast abscess. The review showed some cases of axillary lymph nodes. ${ }^{15}$ Pain is the most common constitutional symptom. Typically, its manifestation is a noncyclical mastalgia, that is, not linked to the menstrual cycle (as in fibrocystic disease, periductal mastitis, or breast abscess). Comparatively, localized pain is very rare in breast cancer. ${ }^{24}$

The protean presentation of breast TB leads to significant diagnostic delay. This is compounded by multiple possible differential diagnoses, especially in resource-limited settings. The main differential diagnoses to be considered are fibroadenoma; breast cancer; inflammatory diseases, such as idiopathic granulomatous mastitis, sarcoidosis, Wegener's granulomatosis, and giant cell arteritis. ${ }^{4,24-26}$ We could also added other infectious diseases, such as brucellosis, actinomycosis, mycotic infections, and fat necrosis. ${ }^{22,25}$ Using PCR or culture as standards by default, no test has satisfactory sensitivity and limited specificity. Of the M. tuberculosis detection methods, microscopy with AFB has, probably, the best balance between sensitivity ( $\sim 80 \%)$ and specificity $(35 \%-65 \%)$. Biopsy has the best sensitivity ( $90 \%$ or more) but very low specificity, whereas FNAC has low sensitivity and specificity. ${ }^{15}$ The culture could be positive only in about one in four cases, and PCR in just over half. ${ }^{15}$ By contrast, in various series in most cases, the diagnosis was made based on pathology and confirmed ex Juventus as they responded to anti-TB therapy. ${ }^{7,27}$ Therefore, generally, pathological examination appears to be of more practical use than bacteriology, especially considering that both culture and PCR are technically challenging and cannot be applied in many settings in low and middle-income countries (LMICs). GeneXpert has not been tested on breast TB as a Cochrane systematic review and meta-analysis of the use of GeneXpert on extrapulmonary TB found variable sensitivities and specificities against culture depending on the type of specimen. When tested on lymph nodes, sensitivity and specificity ranged widely $(56 \%-100 \%$ and $39 \%-100 \%$, respectively); pooled sensitivity and specificity (95\% credible intervals) were $87.6 \%$ (range $81.7 \%-92.0 \%$ ) and $86.0 \%$ (range $78.4-91.5 \%$ ), respectively. ${ }^{27}$ Breast TB overall has a good prognosis. No specific guidelines are available for chemotherapy of breast TB. The most common approach is the standard TB treatment with 2 months of isoniazid, rifampicin, pyrazinamide, and ethambutol, followed by 4 months of isoniazid and rifampicin. ${ }^{14,15}$ Some authors prefer the 9month regimen (2 months of isoniazid, rifampicin, pyrazinamide, and ethambutol, and 7 months of isoniazid and rifampicin) because of a lower relapse rate in general. ${ }^{7}$ We finally do the same because of lake recovery of patient. But sometimes infection with multidrug-resistant TB has been reported. ${ }^{14,27}$ Some authors proposed the continuation 
phase to be extended, commonly to 12 months, but up to 18 months in cases with slow clinical response. We performed a 9 months treatment to obtained complete resolution. In general, complete resolution is obtained in most patients. ${ }^{3,7}$ Some cases have required radical surgical treatment (subtotal or total mastectomy) and minority of patients needed a combination of minor surgical procedures such as cold abscess aspiration and resection of sinus formation or necrotic tissue. ${ }^{15}$ Of all surgical cases underwent lump excision however in many cases, these procedures were performed as an initial step during the workup of the patient diagnosis, and as a consequence, they cannot be fully intentionally included in the treatment plan. ${ }^{7,10}$ The first study which have attempts to review prevalence, risk factors, symptoms, diagnosis, and therapy of breast TB is a systematic manner done by Quaglio et al. ${ }^{15}$ Ideally, data should capture information on possible risk factors, symptoms, and clinical presentations for a better differential diagnosis with other breast diseases, mainly granulomatous mastitis and carcinoma.

\section{CONCLUSION}

In low and middle-income countries as Côte d'Ivoire, breast TB remains a rare and overlooked disease. The reported case showed the difficulties to diagnose and manage a breast TB but the disease should be considered when young patients present with a palpable lump with axillary lymphadenopathy, especially in endemic regions. Although, imaging results were non-specific, they might aid in guiding clinicians. However, confirmation of a diagnosis of TB usually requires an excision biopsy providing histological or bacteriological evidence of infection.

Funding: No funding sources

Conflict of interest: None declared

Ethical approval: Not required

\section{REFERENCES}

1. Nalini B, Vinayak S. Tuberculosis in ear, nose, and throat practice: its presentation and diagnosis. Am J Otolaryngol. 2006;27(1):39-45.

2. World Health Organisation. 2018. Global Tuberculosis Report. Available at: https://www.who.int/tb/publications/global_report/en /. Accessed January 17, 2019.

3. Baharoon S. Tuberculosis of the breast. Ann Thorac Med. 2008;3(3):110-4.

4. Korkut E, Akcay MN, Karadeniz E, Subasi ID, Gursan N. Granulomatous Mastitis: A Ten-Year Experience at a University Hospital. Eurasian J Med. 2015;47(3):165-73.

5. Marinopoulos S, Lourantou D, Gatzionis T, Dimitrakakis C, Papaspyrou I, Antsaklis A. Breast tuberculosis: Diagnosis, management and treatment. Int J Surg Case Rep. 2012;3(11):548-50.

6. Seo HR, Na KY, Yim HE, Kim TH, Kang DK, Oh KK et al. Differential diagnosis in idiopathic granulomatous mastitis and tuberculous mastitis. J Breast Cancer. 2012;15(1):111-8.

7. Khan MR, Barua A, Tarek N, Rouf A, Karim A, Bhiyan NH et al. Mammary tuberculosis: a clinical experience on 50 cases. Chattagram Maa-O-Shishu Hosp Med Coll J. 2014;13:42-4.

8. De Sousa R, Patil R. Breast tuberculosis or granulomatous mastitis: a diagnostic dilemma. Ann Trop Med Public Health. 2011;4:122-5.

9. Meerkotter D, Spiegel K, Page-Shipp LS. Imaging of tuberculosis of the breast: 21 cases and a review of the literature. J Med Imaging Radiat Oncol. 2011;55(5):453-60.

10. Paz MEP, Piazze LR, Garcia FB, SantaCruz E, Palao DC. Mastitis chronica granulomatosa tuberculosa, Diagnóstico y tratamiento en 28 casos. Rev Senol Patol Mamar. 2014;27:27-33.

11. Da Silva BB, Lopes-Costa PV, Pires CG, PereiraFilho JD, Dos Santos AR. Tuberculosis of the breast: analysis of 20 cases and a literature review. Trans R Soc Trop Med Hyg. 2009;103(6):559-63.

12. World Health Organization, "Global Tuberculosis Report 2019”. Available at: https://www.who.int/publications/i/item/9789241565 714. Accessed on 7 June 2020.

13. Yanamandra U, Pathak N, Naithani N, Grover N, Nair V. Tuberculosis of breast: unusual manifestation of tuberculosis. J Infect Chemother. 2012;18(1):109-11.

14. Giri VP, Giri P, Kumawat P. Primary multidrugresistant tuberculosis of the breast-a rare presentation. Ann Med Health Sci Res. 2017;7:70-2.

15. Quaglio G, Pizzol D, Isaakidis P, Bortolani A, Tognon F, Marotta C et al. Breast Tuberculosis in Women: A Systematic Review. Am J Trop Med Hyg. 2019;101(1):12-21.

16. Quaglio G, Pizzol D, Bortolani A, Manenti F, Isaakidis P, Putoto G. Breast tuberculosis in men: A systematic review. PLoS One. 2018;13(4):e0194766.

17. Mathad JS, Gupta A. Tuberculosis in pregnant and postpartum women: epidemiology, management, and research gaps. Clin Infect Dis. 2012;55(11):1532-49.

18. Sharma SK, Kadhiravan T, Banga A, Goyal T, Bhatia I, Saha PK. Spectrum of clinical disease in a series of 135 hospitalised HIV-infected patients from north India. BMC Infect Dis. 2004;4:52.

19. Corbett EL, Steketee RW, ter Kuile FO, Latif AS, Kamali A, Hayes RJ. HIV-1/AIDS and the control of other infectious diseases in Africa. Lancet. 2002;359(9324):2177-87.

20. Wani I, Lone AM, Malik R, Wani KA, Wani RA, Hussain I, Thakur N, Snabel V. Secondary tuberculosis of breast: case report. ISRN Surg. 2011;2011:529368.

21. Meggiorini ML, Vitolo D, Russo A, Trinchieri V, De Felice C. Breast tuberculosis: rare but still present in Italy--a case of mycobacterium breast infection. Breast Dis. 2012;33(4):177-82.

22. Kataria SP, Sharma J, Singh G, Kumar S, Malik S, Kumar V. Primary breast mucormycosis: FNAC 
diagnosis of a rare entity. Diagn Cytopathol. 2016;44(9):761-3.

23. Ruiz-Moreno JL, Peña-Santos G. Bilateral tuberculous mastitis nulliparous patient, initially treated as idiopathic granulomatous mastitis. Ginecol Obstet Mex. 2012;80(3):228-31.

24. Mathew M, Siwawa P, Misra S. Idiopathic granulomatous mastitis: an inflammatory breast condition with review of the literature. BMJ Case Rep. 2015;2015:bcr2014208086.

25. Olu-Eddo AN, Ugiagbe EE. Benign breast lesions in an African population: A 25-year histopathological review of 1864 cases. Niger Med J. 2011;52(4):2116.
26. Amin AL, Purdy AC, Mattingly JD, Kong AL, Termuhlen PM. Benign breast disease. Surg Clin North Am. 2013;93(2):299-308.

27. Kohli M, Schiller I, Dendukuri N, Dheda K, Denkinger CM, Schumacher SG, Steingart KR. $\mathrm{Xpert}^{\circledR} \mathrm{MTB} / \mathrm{RIF}$ assay for extrapulmonary tuberculosis and rifampicin resistance. Cochrane Database Syst Rev. 2018;8(8):CD012768.

Cite this article as: Mian DB, Loué VAS, Angoi AV, Yao A, Kouyate M, N'guessan K et al. Breast location of tuberculosis: a case report and literature review. Int J Reprod Contracept Obstet Gynecol 2022;11:919-23. 